\title{
Efectividad del uso tópico de Salvia officinalis en la disminución del índice gingival en sujetos con gingivitis
}

\author{
Effectiveness of topical use of Salvia officinalis in the gingival index decrease in \\ subjects with gingivitis
}

Valenzuela Melgarejo R¹, Ibieta Hillerns C ${ }^{2}$, Narváez CG 3

\begin{abstract}
RESUMEN
Introducción: La gingivitis es el problema periodontal de mayor prevalencia en la población, por lo que deben buscarse más alternativas de tratamiento y prevención. Objetivo: Evaluar efecto del uso de Salvia officinalis en disminución del índice gingival modificado (IG) en alumnos de Odontología de la Universidad del Desarrollo diagnosticados con gingivitis, año 2010. Sujetos y Método: Fue realizado un estudio cuantitativo, ciego, experimental del tipo ensayo clínico controlado con placebo, sobre la acción de extracto de $S$. officinalis en dentífrico y colutorios para gingivitis inducida por placa bacteriana, en 33 alumnos de odontología de la Universidad del Desarrollo, Concepción; 13 mujeres y 20 hombres entre 21 y 29 años. Fueron divididos en tres grupos; placebo, colutorio y dentífrico. Luego del inicio del tratamiento fueron citados a los 7,14 y 28 días, finalizando el tratamiento el día 14 Resultados: En el control no se encontró diferencias significativas $(p=0.061)$ la primera semana, mientras que en los grupos con colutorio o dentífrico si hubo diferencias significativas ( $p=0.000$ y $p=0.002$ ). La última semana al retirar el placebo, el IG se mantuvo, por otro lado en los grupos con $S$. officinalis no continuó el descenso. La disminución del IG fue mayor para los grupos colutorio y dentífrico que para el control. Discusión: El uso de $S$. officinalis es efectivo en la disminución del IG. El colutorio y dentífrico logran disminuir con mayor rapidez el IG. El colutorio logró la mayor disminución del IG.
\end{abstract}

Rev. Clin. Periodoncia Implantol. Rehabil. Oral Vol. 4(3); 110-113, 2011.

Palabras clave: Salvia officinalis, gingivitis, colutorio, dentífrico, índice gingival.

\section{ABSTRACT}

Background: Gingivitis is the most prevalent periodontal problem in people, so we need to find new alternatives for treatment and prevention. Aim: To evaluate the effects of Salvia officinalis in the declining modified gingival index (GI) in dentistry students of the Universidad del Desarrollo diagnosed with gingivitis, year 2010. Subjects and Methods: This was a quantitative study, blinded, experimental type of placebo-controlled clinical trials on the action of extract of $S$. officinalis in toothpaste and mouthwash for plaque-induced gingivitis in 33 dental students from Universidad del Desarrollo, Concepción; 13 women and 20 men between 21 and 29 years old, were divided into three groups: placebo, mouthwash and toothpaste. After the first appointment we call the patients every 7,14 and 28 days, we ended the treatment at day 14. Results: In the control group there was no significant difference $(p=0.061)$ the first week, while groups with mouthwash and toothpaste, there were significant differences $(p=0.00)$. The last week, as we remove the placebo, the GI was maintained. On the other hand, in groups with S. officinalis the drop did not continue. The decrease of GI was higher in groups with mouthwash and toothpaste than in the control group. Discussion: The use of $S$. officinalis is effective in reducing the GI. The mouthwash and toothpaste decrease more rapidly the IG. The mouthwash achieved the greatest reduction of IG.

Rev. Clin. Periodoncia Implantol. Rehabil. Oral Vol. 4(3); 110-113, 2011.

Key words: Salvia officinalis, gingivitis, mouthwashes, toothpaste, gingival index.

\section{INTRODUCCIÓN}

La gingivitis inducida por placa es la inflamación de la encía producida por bacterias localizadas en el margen gingival(1). Clínicamente detectable por edema, eritema, cambios morfológicos, exudado y sangrado ${ }^{(2)}$. Está presente en gran parte de la población y si no es controlada a tiempo se puede producir la destrucción de los tejidos originando periodontitis y la futura pérdida de las piezas dentales ${ }^{(3)}$. La forma de enfrentar esta enfermedad es mediante el uso de técnicas de cepillado y uso de auxiliares para la higiene oral(4) como auxiliares químicos, algunos son enjuagues como el triclosan y la clorhexidina(5). Esta última tiene efectos colaterales como tinción dentaria y en dorso de lengua, alteración del gusto y erosión de la mucosa, lo que limita su uso en odontología preventiva ${ }^{(4)}$.

Recientemente se han vuelto a valorar las terapias naturales teniendo cada vez más auge la medicina alternativa, adquiriendo relevancia la medicina botánica que se basa en el uso de plantas y sus constituyentes. Aunque el efecto de las plantas curativas pareciera lento son mejores a largo plazo(6).

La Salvia officinalis posee propiedades: antisudorífica, emenagoga, tonificante, antiespasmódica, colerética, hipoglucemiante, estimulante, astringente y antiséptica, estas dos últimas permiten combatir afecciones bucofaríngeas como las aftas bucales, amigdalitis, faringitis y gingivitis ${ }^{(7)}$, pero disminuyendo las posibilidades de enfrentar efectos secundarios que se producirían al utilizar fármacos convencionales.

En el estudio in Vitro realizado por Bozin utilizando aceites esenciales de $S$. officinalis, se comprobaron las propiedades antimicrobianas en Escherichia coli, Salmonella typha, Salmonella enteritidis y Shigella sonei. Además se demostró su actividad antifúngica y antioxidante ${ }^{(8)}$. Horiuchi, obtuvo un extracto de acetona, el cual presentó poca actividad antimicrobiana, luego se purificó el compuesto activo, el que se identificó como ácido Oleoneico y otros triterpenoides mostrando mayor actividad frente a E. coli, P. aeruginosa y S. marcescens ${ }^{(9)}$. Por otro lado Mitic-Culafic, compara el potencial antibacteriano entre el aceite esencial de Salvia y sus componentes fraccionados concluyendo que ambas poseen actividad antibacteriana para $B$. subtilis, $S$. aureus y $E$. coli(10). En el estudio realizado por Özan, se demostró que el extracto de $S$. officinalis, se puede utilizar como medio de transporte para piezas avulcionadas, pero no solo por mantener vivas las células del ligamento periodontal, sino que también poseía actividad antimicrobiana, antiinflamatoria y antioxidante, esta última propiedad atribuida a sus componentes fenólicos ${ }^{(11)}$. La investigación de Anackov, comparan las relaciones taxonómicas de las especies $S$. pratensis y $S$. bertolonii consideradas subespecies de Pratensis. Ambas usadas en la medicina tradicional como alternativa a $S$. officinalis en los resultados del estudio se encuentran diferencias a nivel micromorfologicas en sus hojas como

1. Licenciado en Odontología. Facultad de Odontología, Universidad del Desarrollo. Concepción, Chile.

2. Cirujano Dentista Especialista en Periodoncia. Facultad de Odontología, Universidad del Desarrollo. Concepción, Chile.

3. Licenciada en Bioquímica. Mgs. en Educación Superior. Facultad de Odontología, Universidad del Desarrollo. Concepción, Chile.

Correspondencia autor: Ricardo Valenzuela Melgarejo. rivalenzuelam@gmail.com. Facultad de Odontología, Universidad del Desarrollo. Concepción, Chile. Trabajo recibido el 30/07/2011. Aprobado para su publicación el 30/11/2011. 
en los componentes de sus aceites esenciales, perteneciendo estas a las de categoría de pobres en aceite, mientras que la $S$. officinalis, S. balsamica (miller) y S. griega (triloba), corresponden a las de alto contenido en aceite ${ }^{(12)}$. En resultados obtenidos por Mimica-Dukic, se determinó que las plantas pertenecientes a la familia Lamiacea contienen aceites esenciales con mayor eficiencia antimicrobiana, reconociendo, además, a las plantas fenólicas como el antioxidante natural mas poderoso. Dos plantas aromáticas clasificadas en esta familia fueron las primeras reconocidas como los antioxidantes naturales más potentes: S. officinalis y S. Rosemary ${ }^{(13)}$. Por otro lado Horiuchi, describe como compuesto activo el carnosol, el cual no posee actividad antibacteriana de por si, pero puede potenciar la actividad de otras sustancias que si poseen esta actividad como los aminoglucósidos ${ }^{(14)}$.

Es por ello que en este estudio se pretendió evaluar el efecto del uso tópico de $S$. officinalis en la disminución del índice gingival en alumnos de Odontología de la Universidad del Desarrollo diagnosticados con gingivitis, año 2010.

Permitiendo finalmente concretar si esta planta puede ser utilizada en la práctica clínica, aportando una solución natural y con menos efectos adversos que otros métodos químicos.

\section{MATERIALES Y MÉTODOS}

Se realizó un ensayo clínico controlado.

Los sujetos para el estudio fueron alumnos de Odontología de la Universidad del Desarrollo, Concepción diagnosticados en la clínica odontológica de la Universidad del Desarrollo con gingivitis. Se evaluaron alumnos voluntarios que desearon participar en el estudio y mediante muestreo aleatorio simple se seleccionaron 33 individuos que cumplieron los criterios de inclusión y exclusión y que estaban interesados en participar del estudio. Los criterios para ser incluido en el estudio fueron ser alumno de Odontología de la Universidad del Desarrollo diagnosticado con gingivitis en la clínica odontológica de la Universidad del Desarrollo de 21 a 29 años durante el primer semestre 2010 que presenten gingivitis inducida por placa en cualquiera de sus niveles, sin importar el sexo, que no presenten enfermedades sistémicas, que estén dispuestos a participar del estudio y que hayan firmado el consentimiento informado. Fueron excluidos alumnos que presentaron diabetes, estén bajo tratamiento médico con fármacos, presenten enfermedad psicomotora que dificulte llevar a cabo una correcta técnica de cepillado, alumnos con epilepsia, mujeres que estén amamantando y fumadores.

Los 33 alumnos seleccionados se dividieron, mediante asignación aleatoria, en tres grupos de 11 pacientes cada uno, un grupo para cada tratamiento. La asignación aleatoria se realizó escribiendo el nombre de cada alumno, seleccionando uno al azar y asignándolo al grupo de tratamiento A, luego se realizó lo mismo para el grupo B y $C$ hasta dividir a los 33 . Esto permite lograr grupos de pacientes homogéneos.

La Salvia officinalis deshidratada (Riveros y Cia Limitada, Chillan, Chile) fue procesada en un molinillo para conseguir desmenuzar las hojas y tallos. Con este polvo se preparo el extracto oleoso, el cual consiste en una maceración por una semana de $160 \mathrm{~g}$ de planta por cada $800 \mathrm{ml}$ de aceite vegetal de maíz (chef, Santiago, Chile), luego fue filtrado con gasa para eliminar residuos consiguiendo el extracto oleoso, del cual se utilizó $87.5 \mathrm{ml}$ mezclado con 500 grs de gel neutro (Droguería Alemana, Concepción, Chile) como vehículo y 0.875 grs de ácido ascórbico (Cicarelli, San Lorenzo, Argentina) como preservante para la preparación del dentífrico, que fue envasado en frascos de ungüento de 30 grs (Droguería Alemana, Concepción, Chile) cada uno, los que deben permanecer en un lugar fresco y seco. Éste método original para la preparación del dentífrico es una derivación del método propuesto por Castillo y Martínez ${ }^{(15)}$.

Para la preparación del colutorio, primero se realizó una maceración por 48 horas de 400 grs de planta por cada $1000 \mathrm{ml}$ de etanol al $70 \%$ (Merck S.A., Santiago, Chile), que luego se sometió a filtración por gasa y luego dos veces filtración al vacío para eliminación de residuos, consiguiendo la tintura madre, de la cual se realizó una dilución para lograr un extracto al $50 \%$, diluyendo tintura en agua bidestilada (Sanderson, Santiago, Chile) en iguales proporciones, del cual se utilizó $8 \mathrm{ml}, 4 \mathrm{ml}$ de glicerol (Merck S.A., Santiago, Chile), $988 \mathrm{ml}$ de agua bidestilada y 0.02 grs de ácido ascórbico, que fueron mezclados produciendo una solución color verde en la que al ser refrigerada precipitaron mucílagos (residuos), por lo que se filtro en algodón envuelto en gasa, logrando una solución verde claro sin residuos que fue envasada en frascos de vidrio color ámbar de $200 \mathrm{ml}$ (Droguería Alemana, Concepción, Chile) cada uno. Esta técnica es una derivación del método propuesto por Castillo y Martínez ${ }^{(15)}$.

Finalmente, para el placebo se utilizó $988 \mathrm{ml}$ de agua destilada, 0.01 grs de colorante vegetal menta (Droguería Alemana, Concepción, Chile), $4 \mathrm{ml}$ de glicerol y $0.02 \mathrm{~g}$ de ácido ascórbico, que será envasado de igual forma que el colutorio.

Los alumnos fueron citados para una revisión odontológica voluntaria en la clínica odontológica de la Universidad del Desarrollo, donde se les informo sobre el estudio, se realizó un sondaje para descartar periodontitis y se entrego el consentimiento informado. Luego fueron citados a los siete días para completar la ficha clínica con los datos personales del paciente y se realizó un examen clínico intraoral (día 0) para clasificar su nivel de gingivitis según el índice gingival modificado el cual consiste en puntos que van de 0 a 4 . Siendo el 0 ausencia de inflamación, 1 inflamación leve; con cambio leve de color y textura, pero no de toda la unidad gingival marginal o papilar, 2 inflamación moderada; igual a la anterior, pero abarcando toda la unidad, 3 inflamación moderada; presenta brillo, enrojecimiento y edema de la unidad, y 4 inflamación intensa; en la que hay enrojecimiento intenso, edema, hemorragia espontánea y congestión o ulceración ${ }^{(16)}$. En la ficha clínica se registró el grado más severo del índice gingival de cada sextante, luego se calculo el promedio para cada paciente cada día de revisión. Este índice permite identificar el grado de gingivitis sin realizar sondaje el cual elimina placa, lo que ayudaría al paciente a disminuir la gingivitis, No se realizaron mediciones del índice de placa y no se realizó eliminación mecánica del biofilm por parte del tesista por la misma razón. Además se entregaron los materiales e indicaciones dependiendo de a que grupo de tratamiento corresponden; el grupo A: grupo control, tratado con placebo, se les entregó dos frascos color ámbar de $200 \mathrm{ml}$ cada uno, sin saber que es placebo, para realizar enjuagues con $10 \mathrm{ml}$. (una tapa y media) tres veces al día luego del cepillado, más técnica de cepillado de Bass modificado. Esta técnica fue enseñada por el alumno tesista utilizando macro modelos y un espejo para instruir al paciente sin que queden dudas de la técnica. En ella el cepillo se debe posicionar en $45^{\circ}$ respecto al diente intentando que las cerdas del cepillo entren en el surco gingival para luego realizar un movimiento de atrás hacia adelante y luego hacia la cara oclusal del diente. Ésta se deberá llevar a cabo 3 veces al día durante 14 días.

El grupo B fue tratado con técnica de cepillado de Bass modificado más colutorio de salvia el cual fue entregado en dos frascos color ámbar de $200 \mathrm{ml}$ cada uno, el que se utilizó luego del cepillado dosificando $10 \mathrm{ml}$ (una tapa y media). La solución deberá mantenerse en boca por 60 segundos. $Y$ el grupo $C$ : dentífrico de salvia preparado por el investigador más técnica de cepillado de Bass modificado. Cada presentación deberá ser aplicada vía tópica oral 3 veces al día por 14 días y no ingerir alimentos o bebidas 30 minutos posterior a su aplicación ${ }^{(4)}$.

Los alumnos evaluados según el índice gingival modificado al iniciar el estudio fueron citados para registrar su evolución, utilizando el mismo índice, el día siete y el día catorce. En éste último se indicó finalizar el tratamiento con los extractos y se citó para el día veintiuno, en el que se registró la evolución de los pacientes utilizando sólo el cepillado para el control de la gingivitis. Si bien los alumnos no sabían si utilizaban placebo o extracto en colutorio o dentífrico, el tratante si.

Las experiencias con humanos llevada a cabo en esta investigación estuvieron de acuerdo con las normas éticas de la declaración de Helsinki de 2000, ya que estuvo regida por los principios básicos de toda investigación, protegiendo al paciente, teniendo bases científicas en las que se apoya, fue realizada bajo supervisión de una Cirujano Dentista con especialidad en periodoncia, produjo más beneficios que riesgos para el paciente, los pacientes participaron en forma voluntaria y fueron correctamente informados en todo momento. Todos los pacientes leyeron y firmaron el consentimiento informado. Además los protocolos en la recolección de datos siguen los principios de atención médica en investigación, ya que la atención realizada en el estudio resguardo todos los protocolos sanitarios, va en beneficio del paciente y a ninguno se dejará sin tratamiento convencional para la gingivitis.

Para determinar si existen diferencias significativas entre los valores medios a través del tiempo se utilizó un ANOVA de medidas repetidas, previa comprobación de normalidad con el test de ShapiroWilk y de igualdad de varianzas entre los distintos niveles del factor con la prueba de esfericidad de Mauchly, utilizando el software estadístico SPSS versión 11.5. 


\section{RESULTADOS}

De los 33 pacientes, 13 mujeres y 20 hombres, 2 pertenecientes al grupo control abandonaron el estudio.

Los pacientes al inicio del tratamiento presentaron 2.17 de índice gingival modificado en promedio. Al finalizar el tratamiento, el día 14 , todos los pacientes mostraron una disminución parcial en el índice gingival modificado cercano a 1.02 y a una semana post tratamiento cercano a 0.79 .

El grupo control, al inicio presento un promedio de 2.09 , a los 7 días un promedio de 1.88 , al finalizar el tratamiento 1.73 y 1.70 a una semana del término del tratamiento. Mediante el análisis de medidas repetidas se obtuvo que no hubo diferencias significativas del índice gingival modificado entre el inicio (día 0) y a los 7 días de tratamiento $(p=0.061)$, pero si hubo diferencias significativas a las 2 semanas de tratamiento (día 14) $(p=0.046)$ y a la semana de haber finalizado el tratamiento (día 21) $(p=0.002)$, respecto del día de inicio. Al finalizar el tratamiento con placebo el índice gingival modificado se mantuvo hasta la última revisión $(p=1.00)$ (Figura 1).

El grupo Colutorio presentó al inicio 2.28, a los 7 días 1.78, al finalizar el tratamiento 0.89 y aumento a 1.19 a una semana post tratamiento. Hubo diferencias significativas del índice gingival modificado al comparar todos los tiempos $(p=0.000)$. Al descontinuar el tratamiento con colutorio el índice gingival modificado aumentó (Figura 2).

Finalmente el grupo Dentífrico tuvo una medición inicial de 2.13 , a los 7 días un promedio de 1.75 , al finalizar el tratamiento 0.91 y 1.31 a una semana post tratamiento. Hubo diferencias significativas del índice gingival modificado al comparar el inicio y el día $7(p=0.002)$, entre los 7 días y los 14 días $(\mathrm{p}=0.000)$, entre los 14 días y la última medición $(p=0.001)$. Sin el tratamiento con dentífrico el índice gingival modificado aumentó (Figura 3).

Al comparar, las diferencias del índice gingival modificado entre los días 0 y 14 , de cada grupo, no se encontraron diferencias significativas $(p=0.90)$ entre los dos grupos de aplicación de $S$. officinalis, no así al comparar estos dos con el grupo control, en que si hubo diferencias significativas $(p<0.05)$. Lo mismo ocurrió al comparar las diferencias entre los días 14 (finaliza tratamiento) y 21.

Según el análisis de medidas repetidas existen diferencias significativas de los valores medios a lo largo del tiempo $(p=0.000)$.

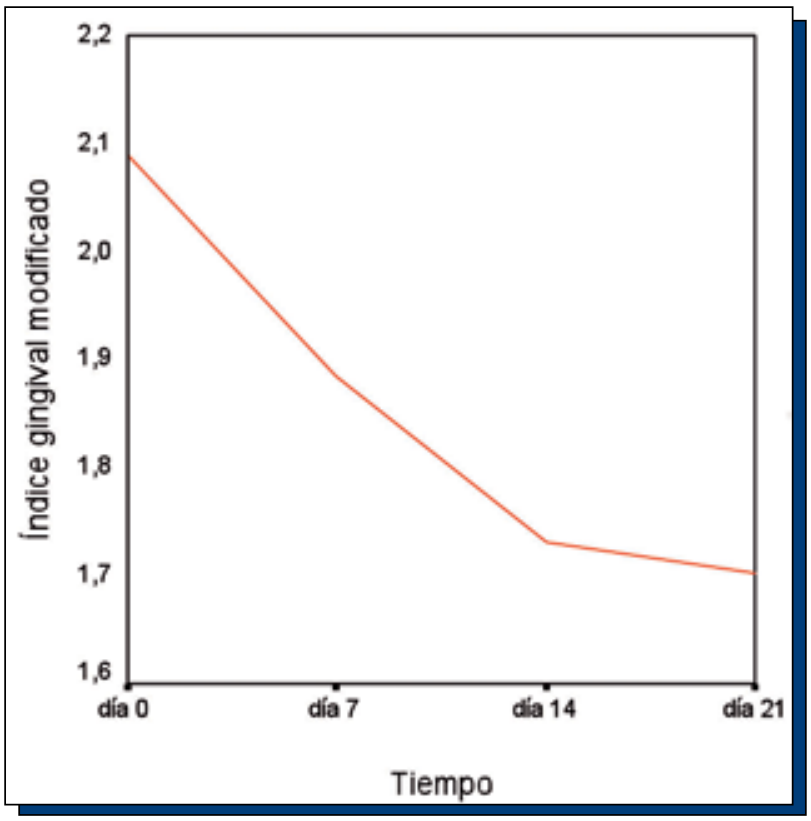

Figura 1. Evolución del índice gingival modificado en el tiempo del grupo control.

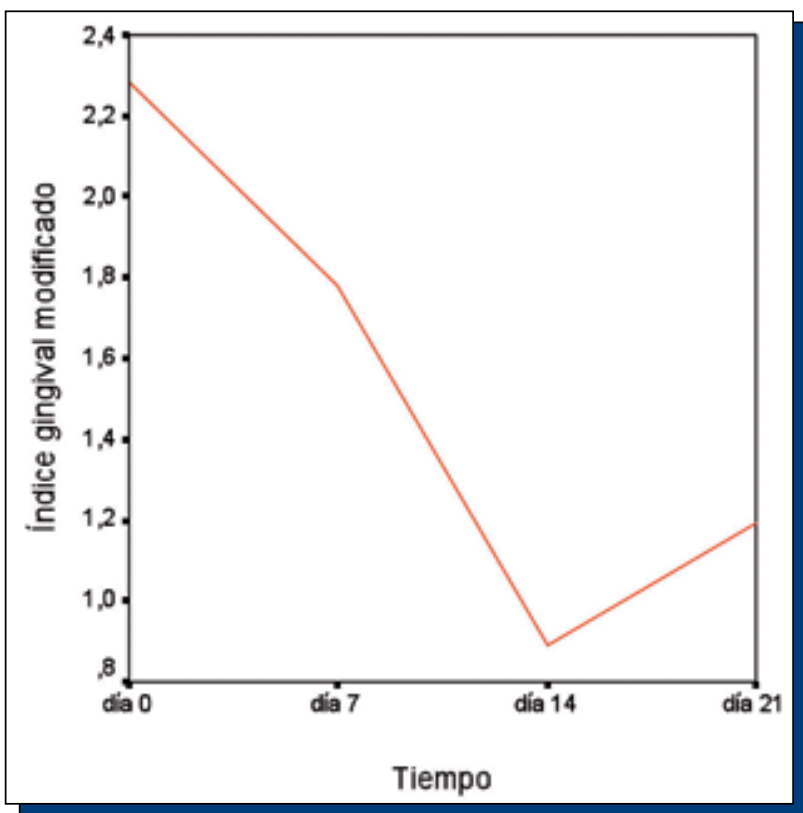

Figura 2. Evolución del índice gingival modificado en el tiempo con la aplicación de colutorio.

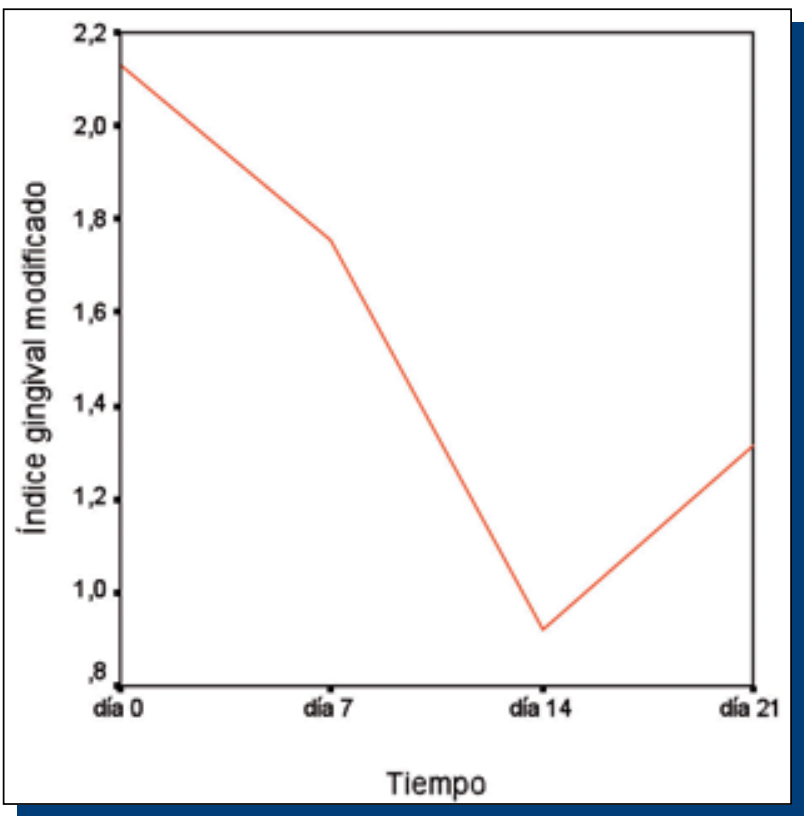

Figura 3. Evolución del índice gingival modificado en el tiempo con la aplicación del dentifrico.

Tabla 1. Medias y Medianas del índice gingival modificado en el tiempo para cada grupo de tratamiento.

\begin{tabular}{|c|c|c|c|c|}
\hline & Día 0 & Día 7 & Día 14 & Día 21 \\
\hline $\mathrm{N}$ & 11 & 11 & 11 & 11 \\
\hline 은 Mediana & 2.33 & 1.83 & 0.83 & 1.3 \\
\hline 彦 Media \pm D.E. & $2.285 \pm 0.394$ & $1.781 \pm 0.312$ & $0.891 \pm 0.226$ & $1.191 \pm 0.177$ \\
\hline $\bar{\jmath}$ ¿े Percentil 25 & 2 & 1.66 & 0.83 & 1 \\
\hline Percentil 75 & 2.58 & 1.915 & 1 & 1.33 \\
\hline $\mathrm{N}$ & 11 & 11 & 11 & 11 \\
\hline Mediana & 2.16 & 1.66 & 0.83 & 1.16 \\
\hline 脫 Media \pm D.E. & $2.131 \pm 0.510$ & $1.754 \pm 0.424$ & $0.919 \pm 0.472$ & $1.314 \pm 0.368$ \\
\hline Фे Percentil 25 & 1.66 & 1.5 & 0.66 & 1 \\
\hline Percentil 75 & 2.58 & 1.995 & 1 & 1.58 \\
\hline $\mathrm{N}$ & 9 & 9 & 9 & 9 \\
\hline _ _ Mediana & 2 & 1.83 & 1.66 & 1.83 \\
\hline Media \pm D.E. & $2.09 \pm 0.363$ & $1.886 \pm 0.465$ & $1.731 \pm 0.629$ & $1.702 \pm 0.455$ \\
\hline ठ̌̉ Percentil 25 & 1.83 & 1.5 & 1.42 & 1.33 \\
\hline Percentil 75 & 2.42 & 2.33 & 2.25 & 2 \\
\hline
\end{tabular}




\section{DISCUSIÓN}

Los resultados de este estudio indican que los pacientes al recibir sólo enseñanzas de técnica de cepillado son capaces de disminuir el índice gingival modificado, como se aprecia en el grupo control (Figura $1)$, pero según el análisis de medidas repetidas no hubo diferencias significativas $(p=0.061)$ entre el índice gingival modificado al inicio del tratamiento y a los 7 días. No así en los grupos a los que además de instruirlos con técnicas de cepillado se les reforzó con la ayuda de colutorio o dentífrico a base de $S$. officinalis, en los que el índice gingival modificado tuvo diferencias significativas $(p=0.000)$ de inmediato la primera semana de uso (Figura 2 y Figura 3 ), por lo que la $S$. officinalis en sus 2 presentaciones ayuda a que el índice gingival modificado disminuya más rápidamente que sólo con técnica de cepillado.

Las diferencias del índice gingival modificado entre el inicio y el final del tratamiento son mayores para el grupo con colutorio, seguido del grupo con dentífrico y finalmente el grupo control, concluyendo que los grupos con colutorio y dentífrico contribuyen a disminuir más el índice gingival modificado que solo con técnica de cepillado.

No hay diferencias significativas al comparar la evolución del índice gingival modificado desde el día 0 al 14 entre los grupos con colutorio y dentífrico, por lo que ambos tendrían igual efecto.

Además se deja en claro que son el colutorio y el dentífrico los que ayudan a disminuir el índice gingival modificado, ya que al retirarlos el índice no continúa descendiendo, observándose un mayor aumento del índice gingival modificado al dejar el dentífrico (Figura 2 y Figura 3).

Al dejar de utilizar el placebo el grupo control no tuvo diferencias significativas $(p=1.000)$ entre el término del tratamiento y la última medición.

En el estudio realizado por Bajaj se demuestra que el uso de clorhexidina en comparación con un colutorio a base de triphala, también una planta que ayuda a combatir la gingivitis, no tienen diferencias en largo plazo, pero el colutorio de triphala al igual que el de salvia se puede utilizar todos los días sin tener que detener su uso, por el contrario el colutorio de clorhexidina debe dejar de utilizarse por los efectos secundarios como tinciones dentarias ${ }^{(17)}$.

Espejel administró un colutorio a base de Echinacea angustifolia 2D, que al igual que el colutorio de salvia, también obtuvo buenos resultados para el control de la gingivitis en 2 semanas de tratamiento ${ }^{(4)}$, demostrando que los colutorios en base a plantas pueden lograr buenos resultados.

Al determinar que en alumnos de Odontología de la Universidad del Desarrollo diagnosticados con gingivitis en la clínica odontológica de la Universidad del Desarrollo el uso tópico de Salvia officinalis, en colutorio y dentífrico, es efectivo en la disminución del índice gingival modificado, se puede plantear comparar, a futuro, el uso de estas presentaciones de $S$. officinalis con dentífricos y colutorios con compuestos de síntesis química, para concluir finalmente cual es la terapia que tiene mayor efectividad en el menor tiempo posible para la prevención y tratamiento de la gingivitis sin los indeseables efectos secundarios.

El estudio fue apoyado por la Universidad del Desarrollo, Concepción. El licenciado Ricardo Valenzuela, la BQ. Carmen Gloria Narváez y la Dra. Carola Ibieta, no tienen ninguna vinculación financiera en relación a los productos involucrados en este estudio. Ricardo Valenzuela es licenciado de Odontología de la Universidad del Desarrollo, Concepción.

\section{CONFLICTOS DE INTERÉS}

Los autores declaran no tener conflictos de interés.

\section{REFERENCIAS BIBLIOGRÁFICAS}

1. Mariotti A. Dental plaque-induced gingival diseases. Annals of periodontology, $1999 \mathrm{Dec}$; 4(1): 11-12.

2. Newman M, Takei G, Henry H, Carranza FA. Carranza Periodontología clínica. 9a ed. México: McGraw-Hill Interamericana Editores; 2004.

3. Lindhe J, Karting T, Lang N. Control mecánico de la placa supragingival. En: Verlag L, editor. Periodontología Clínica e Implantología Odontológica. Madrid: Panamericana; 2003. p. 471-486.

4. Espejel M, Guzmán C, Delgado J. Colutorios de Echinacea angustifolia 2D en el tratamiento de gingivitis simple en niños de 8 a 13 años. Revista ADM, 2006 Nov; 63(6): 205-209.

5. Norero H, López C. Periodoncia: Un desafío diario. $1^{\mathrm{a}}$ ed. Santiago: Arancibia; 2005

6. Pamplona R. Enciclopedia de las plantas medicinales tomo 1. $1^{\text {a }}$ ed. Santiago: Safeliz; 2007.

7. Pamplona R. Plantas para el aparato genital femenino. En: Enciclopedia de las plantas medicinales tomo 2. Santiago: Safeliz; 2007. p. 638-639.

8. Bozin B, Mimica-Dukic N, Samojlik I, Jovin E. Antimicrobial and antioxidant properties of rosemary and sage (Rosmarinus officinalis $L$. and Salvia officinalis L., Lamiaceae) essential oils. J Agric Food Chem, 2007; 55(19): 7879-7885.

9. Horiuchi K, Shiota S, Kuroda T, Hatano T, Yoshida T, Tsuchiya T. Antimicrobial activity of oleanolic acid from Salvia officinalis and related compounds on Vancomycin-Resistant Enterococci (VRE). Bio Pharm Bull, 2007 Jun; 30(6): 1147-1149.
10. Mitic-Culafic D, Vukovic-Gacic B, Knezevic-Vukcevic J, Stankovic S, Simic D. Comparative study on the antibacterial activity of volatiles from sage (Salvia officinalis). Arch Biol Sci, 2005; 57(3): 173-178.

11. Özan F, Akin Z, Tepe B, Er K. Influence of storage media contaigning Salvia officinalis on survival of periodontal ligament cells. The Journal of Contemporary Dental Practice (J Contemp Dent Pract), 2008 Jul 1; 9(5). 12. Anackov G, Bozin B, Zoric L, Vukov D, Mimica-Dukic N, Merkulov L, Igic R, Jovanovic M, Boza P. Chemical composition of essential oil and leaf anatomy of Salvia bertolonii Vis. and Salvia pratensis $L$ (Sect. Plethiosphace, Lamiaceae). Molecules, 2009; 14(1): 1-9.

13. Mimica-Dukic N, Bozin B. Essential oils from lamiaceae species as promising antioxidant and antimicrobial agents. Natural Product Communications, 2007 Ene 31; 2(4): 445-452.

14. Horiuchi K, Shiota S, Kuroda T, Hatano T, Yoshida T, Tsuchiya T. Potentiation of antimicrobial activity of aminoglycosides by carnosol from Salvia officinalis. Bio Pharm Bull, 2007 Feb; 30(2): 287-290.

15. Castillo E, Martínez I. Manual de fitoterapia. Barcelona: ElsevierMasson; 2007.

16. Lobene RR, Weatherford T, Ross NM, Lamm RA, Menaker L. A modified gingival index for use in clinical trials. Clin Prev Dent, 1986; 8(1): 3-6.

17. Bajaj N, Tandon S. The effect of Triphala and Chlorhexidine mouthwash on dental plaque, gingival inflammation, and microbial growth. Int $J$ Ayurveda Res, 2011; 2: 29-33. 